\title{
Depression Screening and Treatment in Uninsured Urban Patients
}

\author{
Margaret A. Meyers, MD, Carla J. Groh, PhD, PMHNP-BC, \\ and Juliann Binienda, PhD
}

Background: Depression has been widely studied in primary care settings, yet studies of medically uninsured populations are lacking. We sought to determine whether depression screening and treatment improved depression scores of a medically uninsured, mostly African American primary care population.

Methods: The study was a prospective repeated-measures design that recruited uninsured patients. Patients were screened for depression, and the rate of depression diagnosis was compared with baseline. Patients who were diagnosed and accepted treatment were randomized to 1 of 4 treatment arms:

(1) usual care; (2) usual care and psychotherapy; (3) usual care and education and psychotherapy; and (4) usual care and education. Patients were then reevaluated at 8, 12, and 24 weeks.

Results: A total of 674 patients participated. Depression prevalence was significantly higher among those screened $(38 \%)$ than at baseline $(16 \%)$. All treatment groups showed a significant reduction in depression scores over a 6-month period, from a mean score of 15 at baseline to 8.3 at 24 weeks $(P<.005)$. All treatment interventions were equally effective.

Conclusion: Screening improves the rate of diagnosis of depression in an uninsured, primarily African American population, and subsequent treatment significantly reduces the burden of depression. (J Am Board Fam Med 2014;27:520-529.)

Keywords: Depression, Practice-based Research, Social Problems, Underserved Populations

Depression, a debilitating disease and one of the most burdensome conditions in the world, is projected to become one of the leading causes of disability by the year $2030 .{ }^{1}$ It is the most prevalent mental health disorder in the United States, with a 12-month prevalence between $7 \%$ and $10 \%{ }^{2}$ and a lifetime prevalence estimated to be $17 \% .^{3}$ Depression is more common among patients with chronic diseases such as diabetes, cardiovascular and cere-

This article was externally peer reviewed.

Submitted 8 September 2013; revised 26 February 2014; accepted 3 March 2014.

From the Mercy Primary Care Center, Detroit, MI (MAM); College of Health Professions, McAuley Health Center, Women's and Gender Studies, University of Detroit Mercy, Detroit, MI (CJG); and Department of Family Medicine and Public Health, Wayne State University, Detroit, MI (JB).

Funding: This study was partially funded by the Blue Cross Blue Shield Foundation of Michigan.

Conflict of interest: none declared.

Corresponding author: Juliann Binienda, $\mathrm{PhD}$, Department of Family Medicine and Public Health, Wayne State University, 3939 Woodward, Room 231, Detroit, MI 48201 (E-mail: jbinien@med.wayne.edu). brovascular disease, cancer, pain, and obesity. ${ }^{4}$ Studies also showed increased risk of diabetes and stroke among people previously diagnosed with depression. ${ }^{5-7}$ In addition, the risk of heart attack among people with depression is more than twice that of people without depression. ${ }^{8}$ The comorbidity of depression and a physical illness adversely affects the outcome of both disorders. ${ }^{9,10}$ Depressed people are more likely to have more physical symptoms, increased functional disability and impairment, poorer adherence to medical treatment and self-care, increased medical costs, and a decreased quality of life compared with the general population. ${ }^{11-13}$ Patients with unrecognized depression consult with their physician more frequently and consume greater health care resources. ${ }^{14,15}$ It also is known that individuals with diagnosed depression are more likely to be uninsured. ${ }^{16,17}$ While studies consistently report that depression is underdiagnosed, ${ }^{18,19}$ underserved and minority populations have a disproportionate burden of depression and resultant disability. ${ }^{20,21}$ 
Although depression can be severely incapacitating, it also is highly treatable if diagnosed. While primary care providers prescribe the majority of antidepressants, ${ }^{21}$ only half of patients with depression in primary care settings are ever diagnosed. ${ }^{22}$ Therefore, the purpose of this research was to examine the effects of formal screening, diagnosis, and treatment of depression in a low-income, urban, uninsured, and predominately African American primary care population.

Our research questions were (1) Does formal screening increase the identification of depression in low-income patients in a primary care setting? (2) Does identification and treatment lower depression scores? and (3) What is the most effective depression intervention for low-income patients in primary care settings?

\section{Methods}

\section{Setting and Sample}

This repeated measures study occurred at a primary care clinic in Detroit, Michigan, over 2 time periods: August 2005 through August 2007 (before electronic medical record use was adopted by the practice) and February 2009 through September 2010 (after electronic medical records were adopted). Gaps in data collection occurred because of funding constraints. We subsequently received a no-cost extension that allowed the study to continue. This free clinic operates Monday through Friday and uses employed rather than volunteer staff. Patients are seen by appointment. The clinic provides primary care, patient-centered medical home services to uninsured, low-income patients ages 18 to 64 years. The center has 2 family physicians and one nurse practitioner handling approximately 5400 annual patient visits.

The inclusion criteria were patients who receive care at the clinic who were not previously diagnosed with depression and/or who were not receiving any form of treatment for depression. History of depression was determined by reviewing the problem list in the patient's chart and by asking the patient if they were ever diagnosed with depression. Approval for the study was obtained from the appropriate institutional review board, and written informed consent was signed by patients before they were enrolled.

\section{Study Protocol}

Establishing a Baseline

Before enrolling patients in the study, the baseline rate of depression diagnosis at the clinic was estimated. An approximate $10 \%$ sample $(n=200)$ of patient medical records from the previous 2 years were randomly chosen and the problem list and notes from the past 5 visits were reviewed for a diagnosis of depression, resulting in a baseline rate of $16 \%$.

\section{Participant Selection}

Patients were recruited while waiting in the examination room by the same research assistant (RA). Recruitment depended on patient flow and the days the RA was available. Patients were excluded if they had been previously approached or if they had a history of mental illness, based on medical chart review or by self-identification. Because of concerns about patient literacy, the consent form was read to the patient by the RA, a nonclinical individual.

\section{Instrument}

Once patients consented to the study, the RA administered the Primary Care Evaluation of Mental Disorders (PRIME-MD) 9-item Patient Health Questionnaire (PHQ-9), a self-report tool that takes approximately 2 to 5 minutes to administer and score. The PHQ-9 provides both diagnostic criteria and a scale for rating the severity of symptoms of depression. It is used as both a screening instrument and a follow-up measure. ${ }^{22}$ It was found to be a valid and reliable instrument when compared with the Medical Outcomes Study Short Form Health Survey. ${ }^{24}$ Patients respond to the 9 items according to the following choices: (a) not at all, (b) several days, (c) more than half the days, or (d) nearly every day. A score of 15 suggests moderate to severe depression and 10 to 14 low severity of depression. In this study, patients with a score of $\geq 5$ were further evaluated by their provider, using clinical judgment, to determine whether they had a diagnosis of depression and to rule out other causes of a positive screen, including bipolar disorder. The cut off score of 5 was used to minimize the chance of a false-negative screen for depression. If the provider determined the patient did not have depression, the patient's role in the study was completed. 


\section{Group Assignment}

Patients diagnosed with depression and who accepted medication were sequentially assigned to 1 of 4 groups: usual care (UC); UC and psychotherapy; UC and education; or UC, psychotherapy, and education. Each consecutive patient was assigned to the next group. The RA followed up with each patient based on the group to which they were assigned, and educational and psychotherapy sessions were scheduled accordingly.

\section{Treatment Groups}

Usual Care

Patients receiving medication had visits with their medical provider as per their provider's regular protocol. Medications were selected at the discretion of the provider based on the patient's profile and comorbidities. The majority of medications prescribed were selective serotonin reuptake inhibitors within the recommended dose ranges. Decisions about medication and dosage changes were made by the provider according to their usual practice.

\section{UC and Psychotherapy}

Patients receiving psychotherapy were scheduled with 1 of 2 therapists at a frequency agreed on by the patient and therapist. The therapists held a $\mathrm{PhD}$ and were experienced psychiatric/mental health nurse practitioners who practice at the site. Cognitive, behavioral, interpersonal, and traumabased psychotherapeutic models of care were used at the discretion of the therapist based on the patient's needs.

\section{UC and Education}

Patients received medication and medical visits similar to the other groups. In addition, they were encouraged to attend a 1-hour-long viewing of a Time Life video (Depression at Time of Diagnosis, C. Everett Koop; Appendix 1) and were given written information on depression developed specifically for the study (Appendix 2).

\section{UC, Psychotherapy, and Education}

Patients received all 3 interventions: UC, psychotherapy, and education.

\section{Reevaluation}

Patients were reevaluated by the psychotherapist, primary care provider, or RA using the PHQ-9 approximately 8,12 , and 24 weeks from the date of initial screening.

\section{Definition of Dependent and Independent Variables}

The outcome/dependent variable in this study was the PHQ-9 depression score. Treatment effectiveness was defined as a statistically significant change in depression score between baseline and the 24th week, with baseline as the reference. The 2 independent variables in the analyses were treatment intervention and time.

\section{Statistical Analyses}

The data were analyzed using the SPSS version 19 statistical software package (IBM, Chicago, IL). A test of proportions was used to determine whether the rate of diagnosing depression with screening is higher than the clinic-estimated baseline of $16 \%$. To determine the effect of the treatment interventions on depression scores, a repeated-measures analysis of variance was performed on the differences of scores from initial screening for the 3 time periods: weeks 8,12 , and 24 . An a priori significance level of $P<.05$ was established.

\section{Results}

A total of 1196 patients were invited to participate, and 674 patients with a mean age of 44 years agreed. Most were African Americans and female, with a high school education or less and a annual household income $<\$ 20,000$. Moreover, $50 \%$ were unemployed (Table 1). Of the 674 who participated, $412(61.1 \%)$ had a PHQ-9 score $\geq 5$ and, upon further clinical evaluation, 255 were diagnosed with depression by their provider. This represents a diagnosis rate of $38 \%$ in patients who were previously undiagnosed in this clinical setting, which was statistically different from the estimated baseline of $16 \%(P<.0001)$ (Figure 1). We dropped 49 people from the study before randomization because they declined medication, wanted to begin counseling at the time of diagnosis, or had other extenuating circumstances.

For those patients who accepted treatment, there was a significant decrease in depression scores from baseline to the 24th week and in the depression scores from one follow-up time to the next, 
Table 1. Sociodemographic Characteristics of the Sample

\begin{tabular}{lc}
\hline Variable & $\begin{array}{c}\text { All Subjects } \\
(\mathrm{n}=674)\end{array}$ \\
\hline $\begin{array}{l}\text { Age (years) } \\
\quad<45\end{array}$ & $314(31.8)$ \\
$\geq 45$ & $360(68.2)$ \\
Race & \\
$\quad$ African American & $641(95.1)$ \\
Other & $33(4.9)$ \\
Sex & \\
$\quad$ Female & $448(66.5)$ \\
Male & $226(33.5)$ \\
Education & \\
High school or less & $432(64)$ \\
College & $242(35.9)$ \\
Income $\geq \$ 20,000$ & $594(88.1)$ \\
Employment & \\
Unemployed & $340(50.4)$ \\
Employed & $334(49.6)$ \\
\hline
\end{tabular}

Data are $\mathrm{n}(\%)$.

except for the scores between the 12th and 24th weeks (Table 2). Of note, the entire sample showed a significant reduction in depression scores over a 6-month period: from a mean score of 15 at baseline to a score of 8.3 at 24 weeks $(P<.005)$.
The analysis of variance results did not show any difference in the effect of the various treatments, using the change in depression scores on the PHQ-9 as the dependent variable (Table 3). For those in the groups who were assigned to psychotherapy, no association between the number of psychotherapy sessions and change in depression scores was detected $(R=0.157 ; P=.138)$.

\section{Discussion}

This study had 3 major findings that merit discussion: (1) The rate of diagnosis of depression significantly increased with screening when compared with regular care. (2) Depression scores decreased with treatment. 3) The degree of score reduction was not dependent on type of treatment.

\section{Screening and Diagnosis Rates}

We demonstrated that using the PHQ-9 to screen for depression resulted in a higher rate of diagnosed depression than standard physician practice. The diagnosis rate increased from 16\% with standard physician practice to $38 \%$ with screening. These results support the recommendations of the US Preventive Services Task Force that routine screening be conducted in offices that have systems

Figure 1. Algorithm showing allocation of participants to treatment groups. PHQ-9, 9-item Patient Health Questionnaire; PCP, primary care physician.




Table 2. Comparison of Depression Scores over the 24-Week Follow-up Period

\begin{tabular}{lcccc}
\hline Paired Differences & Usual Care & $\begin{array}{c}\text { Usual Care and } \\
\text { Psychotherapy }\end{array}$ & $\begin{array}{c}\text { Usual Care and } \\
\text { Education }\end{array}$ & $\begin{array}{c}\text { Usual Care, Psychotherapy, } \\
\text { and Education }\end{array}$ \\
\hline Baseline to 8 weeks & $4.73^{*}$ & $4.55^{\dagger}$ & $6.47^{\dagger}$ & $3.83^{*}$ \\
8 to 12 weeks & 0.30 & 1.00 & 1.09 & 1.52 \\
12 to 24 weeks & 2.15 & 2.49 & -0.16 & 0.61 \\
Baseline to 24 weeks & $5.83^{\dagger}$ & $6.94^{\dagger}$ & $6.35^{\dagger}$ & $5.32^{*}$ \\
\hline
\end{tabular}

*Significant at $P=0.001$.

${ }^{\dagger}$ Significant at $P=0.000$.

in place to ensure accurate diagnosis, effective treatment, and follow-up. ${ }^{25}$

\section{Change in Depression Scores with Treatment}

The entire sample showed a significant reduction in depression scores over a 6-month period: from a mean score of 15 at baseline to 8.3 at 24 weeks $(P<$ $.005)$.

\section{Effect of Different Interventions}

Adding psychotherapy, education, or both to UC did not result in an additional reduction in depression scores. This was an unexpected finding because we anticipated that the combination of medication and therapy would be more effective than medication alone. It could be that the medication had such a great effect that it is difficult to measure any additional benefit from the other interventions (psychotherapy and education). In addition, office visits with providers in UC would likely include therapeutic interactions that were not measured in this study. Moreover, there was no attempt to measure informal therapy that patients may have received from pastors or others in the community. The long-term benefits of psychotherapy and education also may not have been adequately measured in this study since the end point was only 24 weeks.
Even though our study did not find a greater decline in depression scores for those given additional interventions such as psychotherapy and/or education, anecdotal evidence from our patients does indicate that they benefited from both, including increased insight and improved ability to cope with situations. Perhaps the benefits of psychotherapy and education are not measured well by the PHQ-9. Further work to delineate what additional benefits patients derive from psychotherapy/educational sessions needs to be done.

\section{Study Limitations}

The first limitation is the use of a retrospective chart review, which could have resulted in either an under- or overestimation of the diagnosis rate. For example, a lack of documentation on the problem list or notes from the past 5 visits would result in an underestimation. Conversely, patients with depression tend to have more frequent primary care visits for somatic complaints and are therefore more likely to be diagnosed. This may have resulted in an artificially high diagnosis rate. ${ }^{15}$ In addition, the time frame covered by the past 5 visits could vary significantly between patients.

Second, we had a number of patients who did not take advantage of psychotherapy or education

Table 3. Analysis of Variance Comparing Depression Scores in 4 Groups over 24 Weeks

\begin{tabular}{|c|c|c|c|c|c|c|c|c|c|}
\hline & \multicolumn{2}{|c|}{ Usual Care } & \multicolumn{2}{|c|}{$\begin{array}{l}\text { Usual Care and } \\
\text { Psychotherapy }\end{array}$} & \multicolumn{2}{|c|}{$\begin{array}{l}\text { Usual Care and } \\
\text { Education }\end{array}$} & \multicolumn{2}{|c|}{$\begin{array}{c}\text { Usual Care, } \\
\text { Psychotherapy and } \\
\text { Education } \\
\end{array}$} & \multirow[b]{2}{*}{$P$ Value } \\
\hline & Mean (SD) & No. & Mean (SD) & No. & Mean (SD) & No. & Mean (SD) & No. & \\
\hline Baseline & $15.1(4.8)$ & 51 & $14.84(5.2)$ & 51 & $14.3(4.9)$ & 53 & $14.8(4.8)$ & 51 & .849 \\
\hline 8 Weeks & $10.8(7.9)$ & 37 & $11.30(7.6)$ & 33 & $7.3(5.4)$ & 36 & $11.1(6.6)$ & 35 & .052 \\
\hline 12 Weeks & $10.6(8.2)$ & 37 & $11.11(7.9)$ & 37 & $7.2(6.2)$ & 41 & $9.48(5.5)$ & 40 & .074 \\
\hline 24 Weeks & $9.1(8.0)$ & 41 & $8.16(6.7)$ & 31 & $6.9(6.7)$ & 35 & $8.8(6.7)$ & 32 & .567 \\
\hline
\end{tabular}

SD, standard deviation. 
sessions, even though most obvious barriers were removed: the care was free, transportation was provided, and patients already knew and presumably trusted the clinic staff. Contributing factors might include the stigma attached to psychotherapy, which is stronger among African American population $^{26,27}$; the lack of opportunity for patient-provider conversations to help overcome that stigma if they were assigned to the psychotherapy arm; challenges in contacting the patient to schedule the appointment because of disconnected phones; race discordance between the patient and psychotherapist; patient's level of trust in the clinic as a whole; and competing life priorities. Some of the same stressors that contribute to depression (eg, family stressors, poverty) may make it harder to accept or attend treatment. Further work to identify additional barriers and then develop strategies to overcome them needs to be done.

A third limitation was the lack of a control group. Given the significant morbidity associated with depression, there were ethical concerns about identifying individuals with this condition without offering treatment. Because of this, we are unable to identify the level of symptom reduction that might have occurred without treatment.

Generalizability and data collection methods may be other limitations of the study. The findings may not be applicable to the general primary care population because our sample consisted of individuals who were a low-income, uninsured, and largely ethnic minority. There were data points missing because of the difficulties encountered while trying to reach patients for reevaluations. In addition, self-report measures are fraught with methodological issues in terms of data fidelity. Finally, patients who felt they might be depressed may have been more likely to consent to the study.

\section{Conclusions}

To the best of our knowledge this is the first prospective study investigating active depression screening and treatment in an uninsured, predominantly African American population. Screening significantly increased the diagnosis rate. For those who accepted treatment, regardless of treatment modality, depression scores were significantly lower at 24 weeks compared with baseline.

Based on this study, we offer 2 major recommendations. First is a recommendation to conduct additional studies to explore these findings further. For example, a study delineating patient benefits derived from other treatment interventions such as psychotherapy and education should be conducted. These studies need to be longer than 24 weeks. We also recommend studies that look at screening and treatment using methodology including a formal control group and randomization. Other work might include identifying barriers to acceptance of treatment. In addition, work related to the effect of comorbidities such as anxiety and substance abuse on treatment failure could continue.

The most important recommendation, however, is for universal formal depression screening for uninsured, minority, low-income populations, provided means of adequate treatment are available. Based on the results of this study, formal screening and treatment could significantly relieve the burden of depression in one of the most vulnerable populations.

The authors acknowledge Dr. Kendra Schwartz, from Wayne State University School of Medicine, for her assistance in manuscript preparation. We would also like to thank all the staff at Mercy Primary Care Center, especially Dr. Pamela Williams and Ms. Joyce Jordan-Shaw for their hard work in making this project come to life and Dr. Joan Urbancic for helping with its inception and development.

\section{References}

1. Kessler RC, ChiuWT, Demler O, Merikangas KR, Walters EE. Prevalence, severity, and comorbidity of 12-month DSM-IV disorders in the National Comorbidity Survey Replication. Arch Gen Psychiatry 2005;62:617-27.

2. Nimalasuriya K, Compton MT, Guillory VJ. Screening adults for depression in primary care: a position statement of the American College of Preventive Medicine. J Fam Pract 2009;58:535-8.

3. Janosky JE, South-Paul JE, Lin CJ. Pain and depression in a cohort of underserved community dwelling primary care patients. J Am Board Fam Med 2012;25:300-7.

4. Evans DL, Charney DS, Lewis L, et al. Mood disorders in the medically ill: scientific review and recommendations. Biol Psychiatry 2005;58:175-89.

5. Chapman DP, Perry GS, Strine TW. The vital link between chronic disease and depressive disorders. Prev Chronic Dis 2005;2(1):A14.

6. Golden SH, Williams JE, Ford DE, et al. Depressive symptoms and the risk of type 2 diabetes: the Atherosclerosis Risk in Communities study. Diabetes Care 2004;27:429-35.

7. Pan A, Sun Q, Okereke OI, Rexrode KM, Hu FB. Depression and risk of stroke morbidity and mortality: a meta-analysis and systematic review. JAMA 2011;306:1241-9. 
8. Blumenthal JA. Depression and coronary heart disease: association and implications for treatment. Cleve Clin J Med 2008;75(Suppl 2):S48-53.

9. Lett HS, Blumenthal JA, Babyak MA, et al. Depression as a risk factor for coronary artery disease: evidence, mechanisms, and treatment. Psychosom Med 2004;66:305-15.

10. Bowser DM, Utz S, Glick D, Harmon R. A systematic review of the relationship of diabetes mellitus, depression, and missed appointments in a low-income uninsured population. Arch Psychiatr Nurs 2010;24:317-29.

11. Katon W, Ciechanowski P. Impact of major depression on chronic medical illness. J Psychosom Res 2002;53:859-63.

12. Stewart AL, Greenfield S, Hays RD, et al. Functional status and well-being of patients with chronic conditions. Results from the Medical Outcomes Study. JAMA 1989;262:907-13.

13. Kroenke K, Spitzer RL, Williams JBW. The PHQ-9: validity of a brief depression severity measure. J Gen Intern Med 2001;16:606-13.

14. Simon GE, Chisholm D, Treglia M, Bushnell D. Course of depression, health services costs, and work productivity in an international primary care study. Gen Hosp Psychiatry 2002;24:328-35.

15. Blumberg LJ, Nichols LM. The health status of workers who decline employer-sponsored insurance. Health Aff (Millwood) 2001;20:180-7.

16. Emptage NP, Sturm R, Robinson RL. Depression and comorbid pain as predictors of disability, employment, insurance status, and health care costs. Psychiatr Serv 2005;56:468-74.

17. Harrison DL, Miller M J, Schmitt MR, Touchet BK. Variations in the probability of depression screening at community-based physician practice visits. Prim Care Companion J Clin Psychiatry 2010;12. pii: PCC.09m00911.

18. Tylee A, Gandhi P. The importance of somatic symptoms in depression in primary care. Prim Care Companion J Clin Psychiatry 2005;7:167-76.

19. Bailey RK, Patel M, Barker NC, Ali S, Jabeen SJ. Major depressive disorder in the African American population. J Natl Med Assoc 2011;103:548-57.

20. González HM, Tarraf W, Whitfield KE, Vega WA. The epidemiology of major depression and ethnicity in the United States. J Psychiatr Res 2010;44:1043-51.

21. Mark TL, Levit KR, Buck JA. Datapoints: psychotropic drug prescriptions by medical specialty. Psychiatr Serv 2009;60:1167.

22. Olfson M, Tobin JN, Cassells A, Weissman M. Improving the detection of drug abuse, alcohol abuse, and depression in community health centers. J Health Care Poor Underserved 2003;14:386-402.

23. Spitzer RL, Kroenke K, Williams JB. Validation and utility of a self-report version of the PRIMEMD: the PHQ primary care study. Primary care evaluation of mental disorder. JAMA 1999;282: $1737-44$.

24. Nease DE, Maloin JM. Depression screening: a practical strategy. J Fam Pract 2003;52:118-24.

25. U.S. Preventive Services Task Force. Screening for depression in adults: U.S. Preventive Services Task Force Recommendation Statement. Ann Intern Med. 2009;151:784-92.

26. Brown C, Conner KO, Copeland VC, et al. Depression stigma, race, and treatment seeking behavior and attitudes. J Community Psychol 2010;38:350-68.

27. Rusch N, Corrigan PW, Todd AR, Bodenhauser GV. Implicit self-stigma in people with mental illness. J Nerv Ment Dis 2010;198:150-3.

\section{Appendix 1}

Video Program Highlights

\section{REPORT 1: UNDERSTANDING THE DIAGNOSIS}

\section{DEPRESSION}

It is estimated that 1 in 8 people will become clinically depressed at some point in their life. Although it's often called the common cold of mental illness, depression is a serious disorder that requires medical care. It's not a matter of will or a weakness but a psychological condition that can be treated.

\section{SIGNS AND SYMPTOMS}

Loss of interest in things you used to enjoy

Feeling sad for longer than 2 weeks

Lack of appetite or overeating

Insomnia or sleeping more than usual

Aches and pains

Lack of energy or feeling restless

Loss of sexual desire

$\square$ Trouble concentrating or making decisions

Feelings of worthlessness, guilt, or even thoughts of death or suicide

\section{CAUSES}

It is not yet clear to scientist exactly what causes depression. For most people there are several factors, such as:

Inherited susceptibility_having a relative with depression seems to increase the risk

Lack of supportive relationships

Certain personality traits, such as low selfesteem

$\square$ Stress, eg, loss of a spouse or a job 


\section{THE BRAIN AND NEURONS}

Depression may be the result of changes in the chemical communication system that connects the nerve cells, or neurons, in your brain with each other.

$\square$ Neurons communicate by electrical signals and chemicals.

\section{A gap separates 2 neurons}

$\square$ Electrical signals carry a message along each neuron. Chemicals called neurotransmitters carry the message across the gap.

The electrical signal prompts the neuron to release neurotransmitters.

$\square$ Normally, the neurotransmitters dock at receptor sites on the neighboring nerve cell and ignite the electrical signal in that neuron.

$\square$ After the signal is delivered, the neurotransmitters float back to the neuron that sent them. This process is called reuptake.

\section{DEPRESSION}

In depression this communication system between the nerve cells can malfunction.

$\square$ Communication between nerves can break down, for example, because of a lack of certain neurotransmitters or because they fail to dock at their specific sites.

$\square$ New research techniques allow scientists to see changes in the chemistry of the brain and learn more about conditions like depression.

$\square$ The PET scan reveals an image that shows the brain at work. The different colors in the image represent different brain activities.

\section{REPORT 2: WHAT HAPPENS NEXT?}

In most instances, your personal physician can successfully treat your depression as well as other health problems. Your family physician or internist might refer you to a mental health professional such as a:

\section{$\square$ Psychiatrist}

\section{Psychologist}

\section{Social Worker}

Experts say it's probably a good idea to tell people close to you about your illness. Sharing your thoughts and feelings with family members and friends gives them a better chance to help in the recovery process.

Thoughts about harming yourself-even about suicide-should prompt you to see a health professional without delay. Remember, you are not crazy.
You may be feeling the real symptoms of a real medical problem, one that can be relieved with the appropriate treatment.

\section{REPORT 3: TREATMENT AND MAN- AGEMENT}

Depression is usually treated with medication, psychotherapy or a combination of the two. You and your doctor should work together to learn what's right for you.

\section{ANTIDEPRESSANTS}

Drugs called antidepressants can help reestablish the normal path of communication in the brain.

$\square$ One way antidepressants work is by delaying reuptake of the neurotransmitters.

$\square$ This boosts the number of neurotransmitters available and helps carry the signal between nerve cells.

Most people who take antidepressants remark that the medication does not change their personality; it just lets them be who they are when they are not depressed. Antidepressants are not addictive. They don't help everyone, but about 2 out of 3 people benefit. Several weeks of treatment may be necessary before improvement begins. Some people experience side effects, others do not. Make sure to ask your doctor and pharmacist about what to expect when taking mediation. As your symptoms ease, it's important not to reduce your medication without the guidance of your physician.

\section{PSYCHOTHERAPY}

Depression can be effectively treated by several types of psychotherapy (perhaps in combination with medication):

\section{Cognitive therapy}

\section{$\square$ Behavioral therapy}

\section{Interpersonal psychotherapy}

Therapy can be short term or long term, depending on what you want to accomplish. Finding a therapist who is right for you is important. If one person doesn't seem a good match, meet with someone else. Group therapy may also be helpful.

\section{REPORT 4: ISSUES AND ANSWERS}

What are some clues that would help friends and family determine whether a person might be suicidal?

Not everyone who is depressed has thoughts of suicide, but it is important to be aware of the 
problem. Drinking alcohol, being isolated, and feeling helpless are problems that increase the risk of suicide. Ask if the person is feeling suicidal. If they have the idea and a plan for carrying it out, you should get them help right away.

How important is it for a depressed person to have someone to talk to?

Being able to talk with someone helps dispel loneliness and can often help to prevent suicide.

What's the role of exercise?

Exercise such as walking, running, or bike riding can be helpful in dealing with depression. Although a half hour of exercise daily would be beneficial, even 15 minutes every day would be good. Try to do it in the morning in the sunlight or near a window, if you exercise indoors. You should always consult your doctor before beginning an exercise program.

Why do I feel more depressed in winter?

Some people become severely depressed in winter when they are not exposed to enough natural light. This winter depression may be treated by regularly exposing the person to bright light.

What about drugs or alcohol and depression?

Substances abuse can cause symptoms such as hopelessness and lack of control, which can also be caused by depression. So taking drugs or drinking alcohol can make the symptoms of depression worse. A combination of alcohol and some drugs for treating depression could trigger a dangerous interaction.

Sometimes it seems like I'm the only one who feels this way. How can I find out more about other people who feel like me?

Although mental illness is much more recognized and talked about than in the past, it is still difficult to know where to turn for more information. Insightful books about depression and support groups are becoming widespread and should be available in your area.

\section{Appendix 2}

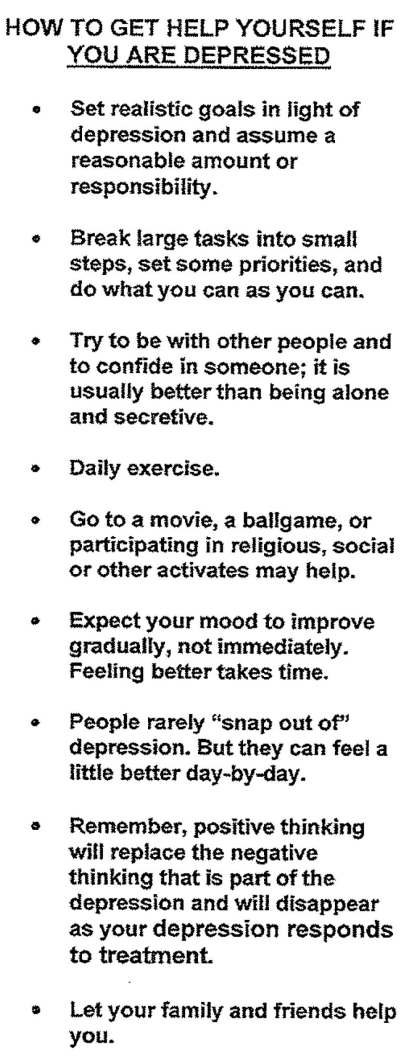

\section{Trinity Health}

MISSION

We serve together in Trinity Health, in the spirit of the Gospel, to heal body, mind, and spirit, to improve the health of our communities, and to steward the resources entrusted to us

Mercy Primary Care Center

5555 Conner Avenue, Suite 2691

Detroit, Michigan 48213

Phone (313) 579-4000

Fax (313) $579-4064$
The Depression Screening

And

Treatment Program

MERCY PRIMARY CARE CENTER

A continuing minisary of Trinity flealth 
- Persistent sad, anxious or empty Mood

- Loss of interest or pleasure in hobbies

- Feelings of hopelessness

- Feelings of guilt, worthlessness, helplessness

- Decreased energy, fatigue, being "slowed down"

- Difficulty concentrating remembering making decisions

- Sleep changes early-morning awakening, frequent nighttime walking or oversleeping

- Weight loss or weight gain

- Thoughts of death or suicide

- Restlessness and irritability

- Persistent physical symptoms that do not respond to treatment, such as headaches, digestive disorders and chronic pain.

Depression is not uncommon; it affects 1 in 10 aduits each year. Women are affected twice as often as men. Depression can occur anytime.

\section{Depression is treatable}

\section{DEPRESSION VS. SADNESS}

How can you distinguish between sadness and depression?

Depression is different from sadness.

It is normal to feel sadness at the death of a loved one, the loss of a job or the ending of a relationship.

Sadness will decrease over time. Depression can last for months, even years.

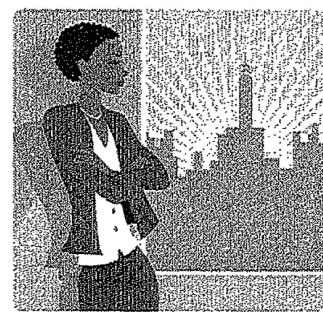

WHAT CAUSES DEPRESSION?

- Imbalances in brain chemistry

- Genetics

- Personality characteristics may place people at risk

- Environmental factors - stress, poverty and violence

- Medical conditions

\section{TREATING DEPRESSION}

Depression can make you feel hopeless and helpless.

Deciding to get treatment can make all the difference.

$80-90 \%$ of depressed people respond to treatment.

\section{TYPE OF TREATMENT}

Medication - Antidepressants are used to correct imbalance in the levels of chemical in the brain. These prescriptions are non habit-forming and anti- depressants have been shown to provide relief in as little as two weeks to three months.

Psychotherapy - Or "talk therapy" is sometimes used alone for treatment of mild depression, or combination with antidepressants for moderate and severe cases.

Education - Learning more about depression can help you control symptoms.

Adapted from APA fact Sheet 